Original

\title{
Evaluación de la seguridad de los anticoagulantes orales de acción directa
}

\author{
Amalia Gassó Sánchez*, José Manuel Real Campaña, José Miguel Franco Sorolla, \\ Bienvenido Javier Povar Marco y Maria Reyes Abad Sazatornil
}

Servicio de Farmacia, Hospital Universitario Miguel Servet, Zaragoza, España

\section{INFORMACIÓN DEL ARTÍCULO}

\section{Historia del artículo:}

Recibido el 2 de octubre de 2018

Aceptado el 11 de octubre de 2018

On-line el $\mathrm{xxx}$

\section{Palabras clave:}

Anticoagulante oral

Hemorragia

Servicio de Urgencias

\begin{abstract}
R E S U M E N
Objetivo: Comparar la incidencia de hemorragias entre los anticoagulantes orales (ACO) y analizar los factores que influyen en la aparición de hemorragias.

Material y métodos: Estudio observacional, retrospectivo unicéntrico. Tras estudiar la población total en tratamiento con ACO, se analizó a los pacientes en tratamiento con un ACO del sector II de Zaragoza que acudieron al Servicio de Urgencias de Hospital Universitario Miguel Servet de julio a diciembre de 2015 por presentar algún evento. Se registraron datos demográficos, variables clínicas y características del evento hemorrágico. Como factores independientes en la aparición de hemorragias se valoró la dosis, fármacos, el sexo y la edad.

Resultados: Había 9.452 pacientes en tratamiento con ACO, de los cuales 371 presentaron un evento hemorrágico (3,9\%). La frecuencia por ACO fue 4,1\% (311) en pacientes tratados con antagonistas de la vitamina $\mathrm{K}(\mathrm{AVK}), 3,8 \%$ (33) con rivaroxaban, 3,3\% (19) con dabigatran y, por último, con apixaban $2,1 \%$ $(8)(p<0,05)$. En el análisis multivariante solo obtuvieron una influencia estadísticamente significativa la selección del anticoagulante y el sexo, en concreto, la dosis de apixaban $2,5 \mathrm{mg}$ y ser mujer presentaban menor riesgo de hemorragia ( $O R=0,1 ; \mathrm{IC}=0,014-0,71$ y $\mathrm{OR}=0,688 ; \mathrm{IC}=0,55-0,85$, respectivamente).

Conclusión: Según los datos obtenidos, las mujeres y los pacientes en tratamiento con apixaban presentaban menor riesgo hemorrágico, si bien existen dudas de si este mejor perfil de seguridad está relacionado con una infradosificación, que podría influir en su efectividad. Por lo tanto, estos resultados deben ser analizados con prudencia y se deben realizar más estudios para confirmar estos datos.
\end{abstract}

(c) 2018 Elsevier España, S.L.U. Todos los derechos reservados.

\section{Safety assessment of direct oral anticoagulants}

\section{A B S T R A C T}

Objective: To compare the occurrence of haemorrhages among the different oral anticoagulants (OAC) and to analyse factors that influence it.

Material and methods: Single-centre, observational, retrospective study. After studying the total population treated with OAC, patients who were treated with an OAC from July 2015 to December 2015 in the II Sector of the Zaragoza Hospital, who consulted the Emergency Department of the Miguel Servet University Hospital and presented a haemorrhagic event, were analysed. Patients' demographic data, clinical variables and data on the haemorrhagic event characteristics were gathered.

Results: There were 9,452 patients treated with an OAC, 371 (3.9\%) of which presented a haemorrhagic event. The frequency per OAC was; 4.1\% (311) in patients treated with vitamin $\mathrm{K}$ antagonists, 3.8\% (33) with rivaroxaban, $3.3 \%$ (19) with dabigatran and $2.1 \%(8)$ with apixaban. In the multivariate analysis, only the choice of anticoagulant and sex had a statistically significant influence of a lower risk of haemorrhage, in particular the dose of apixaban at $2.5 \mathrm{mg}$ and being female. $(\mathrm{OR}=0.1, \mathrm{CI}=0.014-0.71$ and $\mathrm{OR}=0.688$, $\mathrm{CI}=0.55-0.85$, respectively).

\footnotetext{
* Autor para correspondencia.

Correo electrónico: amaliagasso@hotmail.es (A. Gassó Sánchez).
} 
Conclusion: According to the results obtained, females and patients undergoing treatment with apixaban presented lower haemorrhagic risk, although there are doubts about whether this better safety profile is related to underdosing, which could influence its effectiveness. Therefore, these results should be analysed with caution and further studies are needed to confirm this data.

(C) 2018 Elsevier España, S.L.U. All rights reserved.

\section{Introducción}

La hemorragia es uno de los efectos adversos más frecuentes causados por la terapia anticoagulante. La terapia anticoagulante de la que se dispone actualmente se clasifica en terapia anticoagulante parenteral (fondaparinux y heparinas) y oral. Dentro de esta última se encuentran los antagonistas de la vitamina $\mathrm{K}(\mathrm{AVK})$ y los anticoagulantes orales (ACO) de acción directa (ACOD), que incluyen el inhibidor directo de la trombina (dabigatran) e inhibidores directos del factor Xa (rivaroxaban, apixaban y edoxaban $)^{1}$.

Ntaios et al. incluyeron en su metaanálisis 28 estudios de apixaban, dabigatran y rivaroxaban y los compararon con AVK. Los hallazgos confirmaron los principales resultados de los ensayos aleatorizados de los nuevos ACO (ACOD) en entorno real. Así mismo, el metaanálisis de Van der Hulle et al. incluyó 5 estudios y concluyó que, pese a que los ACOD presentaban menor riesgo de hemorragia, el número necesario de pacientes que tratar para prevenir un evento hemorrágico era mayor que en los AVK. A continuación, el metaanálisis de Ruff et al. fue el primero en incluir los ensayos fase III de los ACOD y de mostrar un perfil riesgo/beneficio favorable (menor mortalidad por todas las causas, hemorragias intracraneales incluidas). Por el contrario, mostraron mayor tasa de hemorragias gastrointestinales. Por último, en 2016 se estudió una cohorte de 1.318 pacientes en el registro FANTASII para conocer las características generales y el manejo antiarrítmico en fibrilación auricular no valvular ${ }^{2-9}$.

Los autores del estudio pivotal ARISTOTLE concluyeron que la tasa de hemorragias importantes, de ictus hemorrágicos y de mortalidad por todas las causas fue más baja en el grupo de apixaban respecto a $\operatorname{AVK}(2,1 \text { vs. } 3,0 \% \text {; } p<0,001 \text { y } 3,5 \text { vs. } 3,9 \% \text {; } p=0,047)^{10}$, respectivamente. En el estudio ROCKET AF se obtuvo una frecuencia de sangrado, mayor o menor pero clínicamente relevante, similar entre AVK y rivaroxaban (20,3 vs. $20,7 \%$; $p=0,44)^{11}$ y en el estudio RE-LY se observó menor incidencia de hemorragias mayores en el grupo de pacientes tratados con dosis de dabigatran de $110 \mathrm{mg}$ vs. $\operatorname{AVK}(2,7$ vs. 3,4\%; $p<0,001)$ y hemorragias similares en el grupo en tratamiento con dosis de dabigatran de $150 \mathrm{mg}$ vs. AVK (3,1 vs. $3,4 \% ; \mathrm{p}=0,47)^{12}$.

Recientemente están apareciendo importantes detractores, incluidas algunas sociedades científicas, que publican los sesgos que pueden presentar los ensayos clínicos de los ACOD: es necesario realizar estudios objetivos y en la práctica clínica habitual que permitan avanzar en el conocimiento de estos ACOD y que ayuden a su posicionamiento ${ }^{13}$.

Por ello, se planteó este trabajo, cuyo objetivo fue comparar la incidencia de hemorragias entre los distintos ACO y analizar los factores que influyeron en la aparición de complicaciones hemorrágicas.

\section{Material y métodos}

Se realizó un estudio observacional descriptivo retrospectivo de ámbito hospitalario unicéntrico. En primer lugar, se analizó a todos los pacientes del sector II con edad superior a 18 años tratados con ACO desde el 1 de julio hasta el 31 de diciembre de 2015 (fig. 1).
A continuación, se calculó la incidencia de eventos hemorrágicos en estos pacientes que acudieron al Servicio de Urgencias del Hospital Universitario Miguel Servet. Por último, se comparó la incidencia de hemorragias entre los distintos ACOD (AVK, dabigatran, apixaban o rivaroxaban) y se analizaron los factores influyentes en la aparición de estos eventos.

Los pacientes con evento se seleccionaron a través del programa de gestión de la historia clínica del Servicio de Urgencias y los pacientes con ACO, de la base de datos de receta electrónica. Se incluyó a todos los pacientes en tratamiento con ACO para cualquiera de las indicaciones aprobadas, independientemente de la fecha de inicio del ACO, así como a los que habían presentado cualquier tipo de evento hemorrágico.

Para la población tratada con ACO que presentó un evento hemorrágico se estudiaron variables demográficas (género, edad) y clínicas, con las siguientes escalas: Charlson, HASBLED y $\mathrm{CHADS}_{2}$ VASC $^{14}$. Las 3 escalas aplicadas se estimaron en el momento previo al evento hemorrágico. Por último, también se estudiaron variables relacionadas con el evento hemorrágico (ACO, dosis, alta de urgencias [alta a domicilio, ingreso, fuga o fallecimiento]). Se consideraron dosis bajas de ACOD: apixaban 2,5 mg, dabigatran 75 y $110 \mathrm{mg}$, rivaroxaban 15 y $10 \mathrm{mg}$ y dosis altas: apixaban $5 \mathrm{mg}$, dabigatran $150 \mathrm{mg}$ y rivaroxaban $20 \mathrm{mg}$. Se analizó, además, la localización de la hemorragia. Se agruparon como hemorragias gastrointestinales los siguientes diagnósticos: rectorragias, melenas, hemorragias digestivas, diarreas con sangre y hematemesis.

Por otro lado, en la población global en tratamiento con ACO (con y sin eventos hemorrágicos) se realizó un análisis en función de la edad, género, anticoagulante utilizado y dosis.

En el análisis se distingue el término "paciente» que hace referencia a paciente único, del término "caso", que hace referencia a cada evento hemorrágico que ha tenido el paciente: un mismo paciente puede tener varios casos. Todas las variables salvo los datos demográficos se han calculado sobre los casos, ya que habían podido sufrir modificaciones durante el tiempo transcurrido entre los eventos.

Se ha realizado un análisis descriptivo y un análisis bivariante para explorar la asociación de las distintas variables analizadas con la existencia de hemorragia. En el análisis bivariante, para contrastar las variables cualitativas, se ha aplicado el test de chi-cuadrado. En cuanto a las variables cuantitativas, se ha calculado KolmogorovSmirnov, realizando la comparación de las medias según la prueba de t de Student (distribución normal) o la prueba de U de MannWhitney (distribución no normal).

Por último, para detectar las variables que influyen en la aparición de complicaciones hemorrágicas, se ha realizado un análisis multivariante con modelos de regresión logística y se ha comprobádo la bondad de ajuste de los modelos mediante la prueba de Hosmer-Lemeshow. La estimación del mejor modelo de regresión logística se ha realizado a partir de los posibles factores de riesgo con significación estadística en el análisis bivariante o con especial relevancia clínica, con la intención de analizar la posibilidad de un sesgo de confusión enmascarador del efecto.

Por último, se calculó la incidencia de casos hemorrágicos en la población total en tratamiento con ACO del sector II de Zaragoza. 


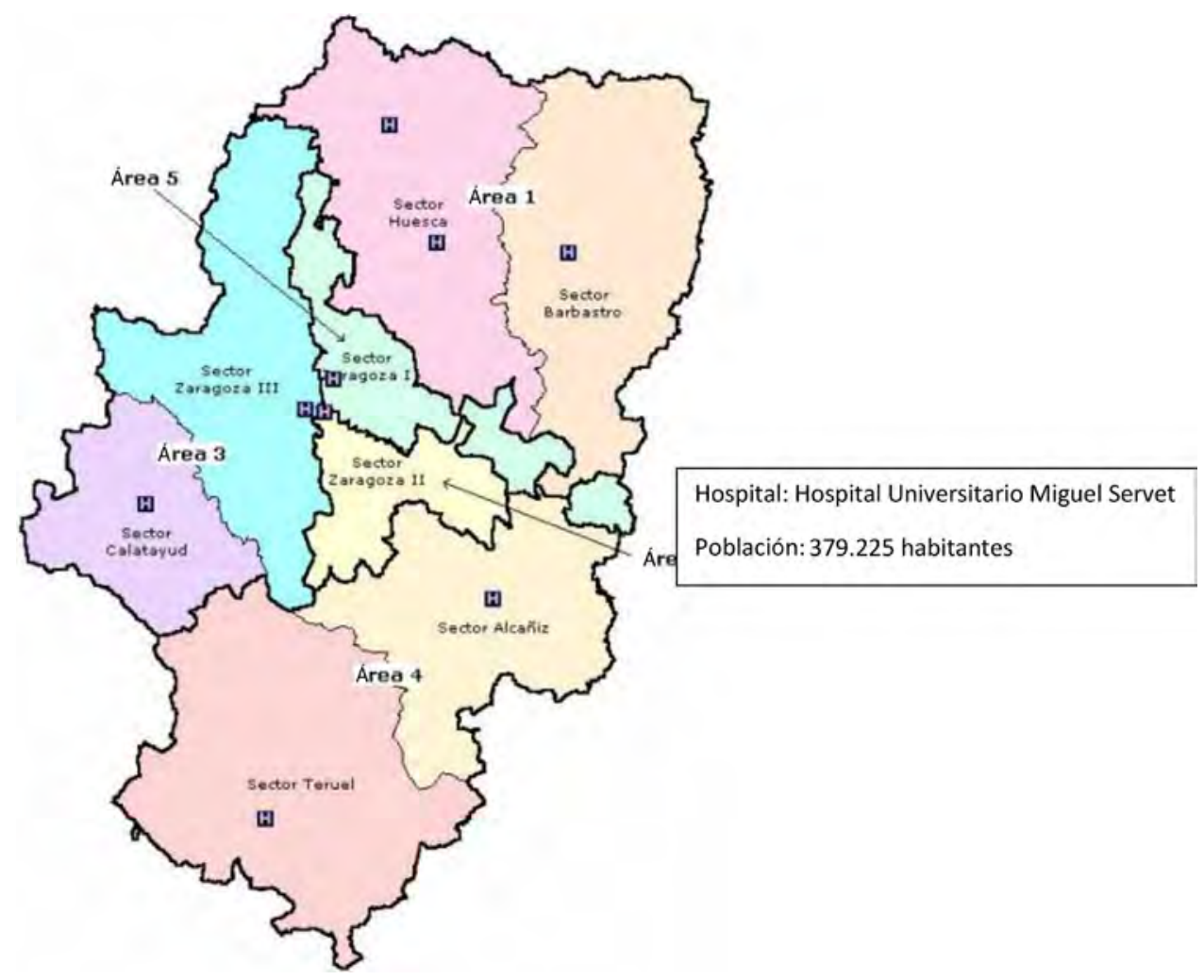

Figura 1. Mapa de los sectores sanitarios de Aragón.

El análisis estadístico se realizó con el programa SPSS Inc. versión 19.0 (licencia de la Universidad de Zaragoza).

Este estudio obtuvo el dictamen favorable del Comité Ético de Aragón (CEICA).

\section{Resultados}

En el sector II de Zaragoza, 9.523 pacientes tomaron ACO en algún momento desde el 1 de julio hasta el 31 de diciembre de 2015. En la tabla 1 se recogen las variables analizadas en esta población. Se encontraron diferencias en la distribución de fármacos prescritos y en la edad $(\mathrm{p}<0,001)$ : se ha observado mayor tasa de prescripción del AVK y de rivaroxaban en pacientes más jóvenes y de apixaban en los de mayor edad.

De los 9.523 pacientes tratados con fármacos ACO, el 3,1\% (299) presentaron algún evento hemorrágico. Destaca que el 19,4\% (47) de estos tuvieron más de un evento hemorrágico, sumando un total de 371 casos hemorrágicos.

Las frecuencias de hemorragias por localización fueron: digestivas $23,2 \%$ (88), seguidas de hematurias $21,8 \%$ (81), epistaxis $13,8 \%$ (51), hemorragias oculares $9,4 \%$ (35) hematomas 6,7\% (25), hemoptisis 5,4\% (20), hemorragias cerebrales 5,4\% (20) úlceras de piel 4,8\% (18), hemorragias bucales 3,3\% (12), hemorragias en heridas $2,7 \%$ (10) y otros tipos de hemorragias 3,0\% (11).

Al diferenciar el número de eventos hemorrágicos por género y edad se obtiene un 3,2\% (150) en mujeres y un 4,6\% (221) en hombres $(\mathrm{p}<0,001)$. Por otro lado, en pacientes menores de 65 años, se obtiene el 33\% (37); entre 65 y 80 años, el 4,3\% (164) y en más de 80 años, el 3,8\% (170) ( $p>0,05)$.

El riesgo hemorrágico se estimó con la escala HASBLED. De todos los casos solo se pudieron calcular un 60,9\%(226). De estos, un 14,3\% (32) tuvieron una puntuación de uno, un 28,9\% (65) de 2, un 32,4\% (73) de 3 y un $24,4 \%$ (55) de 4 . Como se puede observar, la gran parte de los casos tenían un HASBLED entre 3 y 4 (elevado riesgo de hemorragia).

Tras analizar a los pacientes con dosis reducidas de los ACOD, se observa que, de los pacientes con rivaroxaban, un $62,5 \%$ (5) con dosis de $15 \mathrm{mg}$ presentaban insuficiencia renal; con apixaban, el $100 \%$ (2) de los pacientes con dosis de $5 \mathrm{mg} /$ día no presentaban criterios de reducción y en los pacientes con dabigatran ( $150 \mathrm{mg} /$ día y $220 \mathrm{mg} /$ día), en un $5,3 \%$ (1) y un $73,3 \%$ (11) se debió a insuficiencia renal. Esto supone que un 36\% (9) de los pacientes que presentaron hemorragia asociada a ACOD estarían infradosificados.

En la tabla 2 se puede observar la dolencia de los pacientes con evento por la que se inició terapia anticoagulante.

En la tabla 3 se muestra el porcentaje de pacientes que presentaron un evento hemorrágico, según el tipo de fármaco ACO recibido. Se obtuvo relación en la distribución de eventos hemorrágicos con el ACO prescrito $(p=0,011)$. De media, la aparición de hemorragias en el grupo de tratamiento del AVK es $4,1 \%$ y en el grupo en tratamiento con ACOD es de 3,0\% ( $p=0,014)$. Al diferenciar por intensidad de la dosis, se observó que las dosis bajas/intermedias presentaban menor tasa de casos $(\mathrm{p}<0,01)$ respecto a AVK y dosis altas de ACOD.

Se encontraron diferencias estadísticamente significativas $(\mathrm{p}<0,001)$ respecto al cambio de ACO utilizado en el periodo de estudio. Así, en un 9,5\% de los pacientes que presentaron un evento hemorrágico se cambió de ACO, mientras que en los pacientes que no lo presentaron solo se modificó el tratamiento en el 2,1\% de los casos.

Por otro lado, no se encontraron diferencias entre los ACO con respecto al tipo de hemorragia que ocasionaban: en concreto, se encontró una distribución similar en lo que respecta a hemorragias gastrointestinales e intracraneales $(p=0,15)$.

En la tabla 4 se muestra la puntuación obtenida en la escala $\mathrm{CHADS}_{2}$-VASc, la escala Charlson y el riesgo hemorrágico según la escala HASBLED de los pacientes antes de cada evento; se observa 
Tabla 1

Variables recogidas en la población

\begin{tabular}{|c|c|c|}
\hline & Variables recogidas en la población total anticoagulada & Variables recogidas en la población con evento \% (n) \\
\hline \multicolumn{3}{|l|}{ Género } \\
\hline Hombre & $50,5(4.810)$ & $56,5(169)$ \\
\hline \multicolumn{3}{|l|}{ Edad } \\
\hline Media en años & $78,5 \pm 10,9$ & $77,1 \pm 9,8$ \\
\hline \multicolumn{3}{|c|}{ Fármacos y dosis en $\mathrm{mg}$} \\
\hline AVK & $78,8(7504)$ & $83,8(311)$ \\
\hline Rivaroxaban 10 & $0,57(55)$ & - \\
\hline Rivaroxaban 15 & $3,8(362)$ & $2,15(8)$ \\
\hline Rivaroxaban 20 & $5,61(535)$ & $6,73(25)$ \\
\hline Apixaban 2,5 & $2,55(243)$ & $0,53(2)$ \\
\hline Apixaban 5 & $2,28(218)$ & $1,61(6)$ \\
\hline Dabigatran 75 & $0,17(17)$ & $0,26(1)$ \\
\hline Dabigatran 110 & $3,8(362)$ & $4,04(15)$ \\
\hline Dabigtran 150 & $2,23(213)$ & $0,80(3)$ \\
\hline
\end{tabular}

Tabla 2

Enfermedad por la que está indicado el tratamiento anticoagulante en la población con eventos

\begin{tabular}{|c|c|c|c|c|}
\hline Indicación & $\operatorname{AVK} \%(n)$ & Rivaroxaban \% (n) & Apixaban \% (n) & Dabigatran \% (n) \\
\hline Fibrilación auricular no valvular & $67,5(210)$ & $84,8(28)$ & $100(8)$ & $84,2(16)$ \\
\hline TVP & $4,2(13)$ & - & - & - \\
\hline TEP & $5,8(18)$ & $3(1)$ & - & - \\
\hline Prótesis valvular & $20,3(63)$ & $3(1)$ & - & $5,3(1)$ \\
\hline Trombocitosis & $1,6(5)$ & $3(1)$ & - & - \\
\hline IAM & $0,6(2)$ & $6,1(2)$ & - & $10,5(2)$ \\
\hline
\end{tabular}

IAM: infarto agudo de miocardio; TEP: tromboembolia pulmonar; TVP: rrombosis venosa profunda.

Tabla 3

Comparación de las frecuencias de eventos hemorrágicos, hemorragias gastrointestinales y cerebrales en los pacientes según el anticoagulante oral recibido

\begin{tabular}{|c|c|c|c|c|c|c|}
\hline & AVK & Rivaroxaban & Apixaban & Dabigatran & Total & $\mathrm{p}$ \\
\hline N. ${ }^{\circ}$ de casos hemorrágicos & $4,1(311)$ & $3,8(33)$ & $2,1(8)$ & $3,3(19)$ & $3,9(371)$ & $<0,05$ \\
\hline Casos hemorrágicos gastrointestinales \% (n) & $0,9(68)$ & $1,5(13)$ & $0,5(2)$ & $1,4(8)$ & $1,0(91)$ & $>0,05$ \\
\hline Casos hemorrágicos cerebrales \% ( $\mathrm{n}$ ) & $0,3(19)$ & $0(0)$ & $0,3(1)$ & $0(0)$ & $0,2(20)$ & \\
\hline
\end{tabular}

Tabla 4

Puntuación obtenida de las escalas analizadas en la población con eventos (HASBLED, CHADS 2 -VASc y Charlson)

\begin{tabular}{|c|c|c|c|c|c|}
\hline & AVK n (\%) & Rivaroxaban n (\%) & Apixaban n (\%) & Dabigatran n (\%) & $\mathrm{p}<0,05$ \\
\hline \multicolumn{6}{|l|}{ HASBLED } \\
\hline Riesgo bajo & $22(7,07)$ & $4(12,12)$ & $3(37,50)$ & $2(10,5)$ & \\
\hline Riesgo intermedio & $56(18)$ & $6(18,18)$ & $1(12,5)$ & $3(15,78)$ & \\
\hline Riesgo alto & $126(40,51)$ & $1(3,03)$ & $1(12,5)$ & $2(10,52)$ & \\
\hline \multicolumn{6}{|l|}{$C H A D S_{2}-V A S C$} \\
\hline $0^{\mathrm{a}}$ & $1,6(6)$ & & & & \\
\hline $1^{\mathrm{b}}$ & $2,7(10)$ & & & & \\
\hline $2^{c}$ & $10,5(39)$ & & & & \\
\hline$>2^{c}$ & $85,2(315)$ & & & & \\
\hline \multicolumn{6}{|l|}{ Charlson } \\
\hline Sin comorbilidad $(0)$ & $1,0(4)$ & & & & \\
\hline Comorbilidad baja ( 1 y 2 ) & $4,0(15)$ & & & & \\
\hline Comorbilidad alta (3 y 4 ) & $7,3(27)$ & & & & \\
\hline Comorbilidad muy alta $(>5)$ & $87,7(325)$ & & & & \\
\hline
\end{tabular}

a Valor 0: no se recomienda iniciar terapia anticoagulante.

b Valor 1: podría iniciar terapia anticoagulante.

c Valor $>2$ : se recomienda iniciar terapia anticoagulante.

que la mayor parte de los pacientes con alto riesgo hemorrágico (40,5\%; 108) estaban en tratamiento con AVK. Por el contrario, si se comparan solo los pacientes con riesgo hemorrágico bajo, se producen más casos con apixaban 37,5\% (3), seguidos de rivaroxaban $12,1 \%$ (4), dabigatran 10,5\% (2) y, por último, de AVK 7,07\% (21) $(\mathrm{p}<0,05)$.

También se recogió el valor de INR en el momento de la hemorragia en los pacientes con evento: solo se obtuvieron los datos en 245 pacientes (66\%). En los pacientes tratados con AVK, se obtuvo un INR $<2$ en el 13,5\% (42) de los pacientes, con un INR entre 2-3 hubo un $36 \%$ de los pacientes (112) y con INR > 3, un 20,6\% (64). En los pacientes tratados con rivaroxaban, con INR $<2$ hubo un $36,4 \%$ (12) y para INR entre 2-3 hubo un 6,1\% (2). Los pacientes tratados con apixaban tuvieron un INR $<2,62,5 \%$ (5) y, por último, en los pacientes tratados con dabigatran, para un INR $<2$ hubo un $36,8 \%$ (7) y para INR entre $2-3$, hubo un $5,3 \%$ de los pacientes (1).

No se halló una relación entre el tipo de alta en urgencias y el ACO $(p=0,96)$. Sin embargo, se observó una tendencia mayor de ingresos en los pacientes en tratamiento con AVK (29,3\%), seguidos del dabigatran (26,3\%) y del apixaban (25\%): ingresaron en total 
Tabla 5

Variables que explican la existencia de casos hemorrágicos en pacientes en tratamiento con anticoagulantes orales a partir de regresiones logísticas

\begin{tabular}{llll}
\hline & OR & \multicolumn{2}{c}{ IC 95\% para EXP(B) } \\
\cline { 3 - 4 } & & Inferior & Superior \\
\hline $\begin{array}{l}\text { Género (mujer) } \\
\text { Edad }\end{array}$ & $\mathbf{0 , 6 8 8}$ & $\mathbf{0 , 5 5 5}$ & $\mathbf{0 , 8 5 2}$ \\
$\quad<65$ años & & & \\
$>$ 80 años & 0,719 & 0,500 & 1,035 \\
Anticoagulantes orales en $m g$ & & 0,754 & 1,182 \\
Apixaban 5 & 0,944 & & \\
Dabigatran 110 & 0,641 & 0,282 & 1,457 \\
Dabigatran 150 & 0,985 & 0,579 & 1,676 \\
Dabigatran 75 & 0,322 & 0,102 & 1,016 \\
Rivaroxaban 20 & 1,368 & 0,181 & 10,344 \\
Rivaroxaban 15 & 1,043 & 0,680 & 1,602 \\
Rivaroxaban 10 & 0,591 & 0,302 & 1,159 \\
Apixaban 2,5 & 0,000 & 0,000 & 0 \\
\hline
\end{tabular}

Variables de referencia:

Variable dependiente: evento hemorrágico no.

Variables independientes: género hombre; anticoagulante oral antagonista de la vitamina K; edad entre 65 y 80 años.

En negrita, variables significativas $(\mathrm{OR}<1)$.

un 32,4\% (97) de los pacientes. Además, de los 13 pacientes fallecidos, el grupo en tratamiento con apixaban 12,5\% (1) sobrepasa a los otros ACO: un 3,0\% (1) tratados con rivaroxaban; un 5,3\% (1) con dabigatran y un $3,2 \%$ (10) con AVK, pero sin diferencias estadísticamente significativas $(p=0,26)$. Cabe destacar que se observó una relación estadísticamente significativa entre el valor estimado del HASBLED y el fallecimiento ( $\mathrm{p}<0,001)$.

En la tabla 5 se muestra el modelo multivariante de regresión logística realizado. Se halló asociación entre la probabilidad de aparición de un evento hemorrágico y el género $(\mathrm{OR}=0,688$; IC: 0,55-0,85), es decir, el género femenino respecto al masculino tiene menor riesgo de padecer un evento hemorrágico. Así mismo, el apixaban de $2,5 \mathrm{mg}$ tiene menos riesgo de tener un evento respecto al acenocumarol (OR=0,1; IC: 0,014-0,71).

\section{Discusión}

Los pacientes bajo tratamiento con AVK presentan más prevalencia de acontecimientos hemorrágicos que los pacientes bajo tratamiento con ACOD, independientemente de la enfermedad que presentaran los pacientes y dependientemente de la dosis, el fármaco y el género.

Sin embargo, en la regresión logística se obtuvo que solo los pacientes con apixaban de $2,5 \mathrm{mg}$ tenían menor riesgo de presentar un evento que los pacientes con AVK, con diferencias estadísticamente significativas. En cualquier caso, estos resultados deben ser analizados con prudencia debido a que los intervalos de confianza son muy amplios por el escaso número de pacientes disponibles para algunas dosis. Además, la sobreutilización de esta dosis puede suponer una infradosificación de los pacientes y, por tanto, una posible menor efectividad en la prevención del ictus y de la embolia sistémica. De hecho, cabe destacar que, tras analizar a los pacientes que utilizaban dosis bajas de los ACOD, se obtuvo que un tercio de estos estaba siendo infradosificado. Por ello, serán necesarias futuras investigaciones para lograr conclusiones definitivas.

La mayor parte de hemorragias fueron gastrointestinales (melenas, rectorragias, hemorragias digestivas, diarreas con sangre y hematemesis), que superaron a las hemorragias de tipo urinario (24,5 vs. $21,8 \%$ ). Del mismo modo, en el estudio de Guerrouij et al., en el que solo se analizan eventos hemorrágicos en una población con AVK, las hemorragias gastrointestinales superan a las hematurias $(40,8 \text { vs. } 14,1 \%)^{15}$. Al separar las hemorragias gastrointestinales por cada grupo de fármaco anticoagulante recibido, aunque sin hallarse significación estadística, se observó mayor número de estas en el grupo en tratamiento con rivaroxaban y dabigatran que con AVK, lo cual también fue observado por Ruff et al. (OR 0,48; IC: $0,39-0,59)$ y Sherwood et al. (OR 1,25; IC: $1,01-1,55)^{16,17}$.

Respecto a las hemorragias cerebrales, todas se producen con los AVK $(0,25 \%)$, salvo una, que se produce en un paciente con apixaban (0,25\%), aunque el tamaño muestral analizado de pacientes tratados con AVK es muy superior al resto de los ACOD. En cualquier caso, muchos estudios concluyen que la frecuencia de hemorragias intracraneales es menor en el grupo de los ACOD, como Miller et al. (OR 0,49; IC: 0,36-0,66) y Ruff et al. (OR 0,48; IC: 0,39-0,59) ${ }^{17,18}$.

Por otro lado, a pesar de no encontrarse diferencias significativas entre la variable fallecimiento y ACO, se debe tener en cuenta que aún no existen comercializados antídotos específicos para revertir las hemorragias por rivaroxaban o apixaban y que, durante el periodo de estudio, aún no se encontraba disponible idarucizumab para revertir las hemorragias de dabigratran, lo cual obviamente modifica el tratamiento de estas hemorragias. En el estudio DECOVER, en el que se evaluó el grado de acuerdo con método Delphi entre hematólogos y urgenciólogos respecto al manejo de hemorragias, ya se puso de manifiesto la necesidad de antídotos específicos para todos los $\mathrm{NACO}^{19}$.

Se encontró una mortalidad muy superior por hemorragia cerebral que por hemorragia gastrointestinal (30 vs. 7,8\%), al igual que se observó en otros estudios, lo cual confirma que la hemorragia cerebral es un predictor de mortalidad ${ }^{20}$. No se encontraron diferencias estadísticamente significativas entre los ACO analizados en la frecuencia de hemorragias cerebrales. Estos resultados no concuerdan con los resultados obtenidos por Becattini et al. respecto a la mortalidad hallada o a la incidencia de hemorragias cerebrales, que fue mayor en el grupo de los AVK vs. NACOD (51 vs. $21 \%$; IC: $2,59-5,54)^{21}$. Por el contrario, algunos estudios que comparan los anticoagulantes apuntan una mayor seguridad con el apixaban en hemorragias intracraneales respecto al resto de los $\mathrm{ACOD}^{22}$.

En este estudio se obtuvo que, según los valores de la escala HASBLED, un porcentaje elevado de pacientes presentaba riesgo hemorrágico alto, y que existía, además, una asociación significativa entre la mortalidad y el tipo de anticoagulante prescrito $(\mathrm{p}<0,001)$. Además, la mayor parte de los pacientes con alto riesgo hemorrágico estaban en tratamiento con AVK, lo que resulta coherente con el mayor número de casos hemorrágicos, de fallecidos y de ingresos respecto a los pacientes en tratamiento con los otros ACO. Esto indica que los pacientes analizados con casos hemorrágicos en tratamiento con ACOD presentaban riesgos más bajos de hemorragia que con el $\mathrm{AVK}^{23}$.

Por último, se debe tener en cuenta que este estudio presenta algunas limitaciones como es la diferencia en el tiempo de seguimiento de la población analizada, muy inferior en los ACOD con respecto a los AVK. De igual manera, tipos de hemorragias muy infrecuentes solo se detectarán cuando se analizan cohortes de pacientes de un número elevado y con seguimientos largos. Este hecho igualmente podría estar beneficiando al grupo de los ACOD. En cualquier caso, cabe destacar las limitaciones inherentes al tipo de diseño (retrospectivo y unicéntrico) y otras importantes, como la falta de información acerca de la gravedad de la hemorragia o los numerosos datos perdidos de los valores INR en el momento de la hemorragia.

Por último, las escalas utilizadas $\mathrm{CHADS}_{2}$-VASc y HASBLED son para casos de fibrilación auricular, diagnóstico mayoritario en la población estudiada; sin embargo, esta aproximación podría estar sesgando los resultados. Además, al no haber analizado la indicación de la terapia anticoagulante en el total de la población anticoagulada, no se puede realizar un análisis en esta subpoblación concreta de pacientes (solo se dispone de estos datos en la población con eventos). 
En conclusión, es importante verificar que los nuevos anticoagulantes a las dosis utilizadas en la práctica clínica real (en alta proporción dosis inferiores a las recomendadas), aunque presentan menores tasas de hemorragias, no se relacionan con un mayor riesgo embólico.

\section{Conflicto de intereses}

No se declara ningún conflicto de interés.

\section{Bibliografía}

1. Fichas técnica del apixaban, rivaroxaban, dabigatran y edoxaban. Aemps.gob.es. Madrid: Agencia Española del Medicamento [consultado el 30 de marzo de 2016]. Disponible en: http://www.aemps.gob.es.

2. Rivera-Caravaca JM, Marín F, Esteve-Pastor MA, et al. Usefulness of the 2 MACE score to predicts adverse cardiovascular events in patients with atrial fibrillation. Am J Cardiol. 2017;120:2176-81.

3. Caldeira D, Rodrigues FB, Barra M, Santos AT, de Abreu D, Gonçalves N, et al. Nonvitamin $\mathrm{K}$ antagonist oral anticoagulants and major bleeding-related fatality in patients with auricular fibrillation and venous thromboembolism: A systematic review and meta-analysis. Heart. 2015;101:1204-11.

4. Böttger B, Thate-Waschke IM, Bauersachs R, Kohlmann T, Wilke T. Preferences for anticoagulation therapy in auricular fibrillation: The patients' view. J Thromb Thrombolysis. 2015;40:406-15.

5. Ntaios G, Papavasileiou V, Makaritsis K, et al. Real-world setting comparison of nonvitamin-K antagonist oral anticoagulants versus vitamin-k antagonists for stroke prevention in atrial fibrillation: A systematic review and meta-analysis. Stroke. 2017;48:2494-503.

6. Roldan Rabadan I, Anguita Sanchez M, Marin F, et al. Tratamiento antiarrítmico actual de la fibrilación auricular no valvular en España. Datos del registro FANTASIIA. Rev Esp Cardiol. 2016;69:54-60.

7. Van der Hulle T, Kooiman J, den Exter PL, et al. Effectiveness and safety of novel oral anticoagulants as compared with vitamin K antagonists in the treatment of acute symptomatic venous thromboembolism: A systematic review and metaanalysis. J Thromb Haemost. 2014;12:320-8.

8. Moss JD, Cifu AS. ACC/AHA Task Force on Practice Guidelines. Management of anticoagulation in patients with atrial fibrillation. JAMA. 2015;314:291-2.

9. Ganz LI. Epidemiology of and risk factors for auricular fibrillation. Uptodate.com. Philadelphia (PA) Wolters Kluwer Health [consultado el 10 de octubre de 2016]. Disponible en: https://www.uptodate.com

10. Granger CB, Alexander JH, McMurray JJV, Lopes RD, Hylek EM, Hanna M, et al. Apixaban versus warfarin in patients with atrial fibrillation. N Engl J Med. 2011;365:981-92.
11. Lopez-Sendon J, Merino JL. Resultados e implicaciones del estudio ROCKET. Rev Esp Cardiol. 2013;13 Supl C:42-6.

12. Connolly SJ, Ezekowitz MD, Yusuf S, Eikelboom J, Oldgren J, Parekh A, et al. Dabigatran versus warfarin in patients with auricular fibrillation. N Engl J Med. 2009;361:1139-51.

13. Erviti López J. Boletín de información terapéutica de navarra. Pamplona: Servicio Navarro de Salud; 2016 [consultado el 29 de noviembre 2016]. Disponible en: www.bit.navarra.es.

14. Calculadores escala HASBLED y escala CHADS2-VASc. Samiuc.es. Sevilla: Sociedad Andaluza de Medicina Intensiva y Unidades Coronarias [consultado el $20 \mathrm{de}$ septiembre de 2016]. Disponible en: www.samiuc.es.

15. Guerrouij M, Uppal CS, Alklabi A, Douketis JD. The clinical impact of bleeding during oral anticoagulant therapy: Assessment of morbidity, mortality and postbleed anticoagulant management. J Thromb Thrombolysis. 2011;31:419-23.

16. Sherwood MW, Nessel CC, Hellkamp AS, Mahaffey KW, Piccini JP, Suh EY, et al. Gastrointestinal bleeding in patients with atrial fibrillation treated with rivaroxaban or warfarin: ROCKET AF Trial. J Am Coll Cardiol. 2015;66:2271-81.

17. Ruff CT, Giugliano RP, Braunwald E, Hoffman EB, Deenadayalu N, Ezekowitz MD, et al. Comparison of the efficacy and safety of new oral anticoagulants with warfarin in patients with auricular fibrillation: A meta-analysis of randomised trials. Lancet. 2014;383:955-62.

18. Miller CS, Grandi SM, Shimony A, Filion KB, Eisenberg MJ. Meta-analysis of efficacy and safety of new oral anticoagulants (dabigatran, rivaroxaban, apixaban) versus warfarin in patients with auricular fibrillation. Am J Cardiol. 2012;110:453-60.

19. Vicente V, Martin A, Lecumberri R, et al. Perspectivas clínicas del manejo de la hemorragia en el paciente tratado con anticoagulante oral: Estudio DECOVER Emergencias. 2017;29:18-26.

20. Singer AJ, Quinn A, Dasgupta N, Thode H Jr. Management and outcomes of bleeding events in patients in the emergency department taking warfarin or a non-vitamin $\mathrm{K}$ antagonist oral anticoagulant. J Emerg Med [revista en Internet]. 2016 [consultado el 1 de noviembre de 2016]; [aprox. 2 p.]. Disponible en: https://www.ncbi.nlm.nih.gov/pubmed/2779350.

21. Becattini C, Franco L, Beyer-Westendorf J, Masotti L, Nitti C, Vanni S, et al. Major bleeding with vitamin $\mathrm{K}$ antagonists or direct oral anticoagulants in real-life. Int J Cardiol. [revista en Internet*] 2016. [consultado el 1 de noviembre de 2016]; [aprox. 2 p.] Disponible en: https://www.ncbi.nlm.nih.gov/pubmed/27843050.

22. Gonzalez-Bueno J, Sierra-Torres ML, Pérez Blanco JL. Riesgo hemorrágico asociado a los nuevos anticoagulantes orales. Rev OFIL [revista em Internet] 2015; [consultado el 1 de noviembre de 2016]. 25(1): [aprox. 1 p.] Disponible en: http://www.revistadelaofil.org/riesgo-hemorragico-asociado-los-nuevosanticoagulantes-orales/.

23. Lip GY, Keshishian A, Kamble S, Pan X, Mardekian J, Horblyuk R, et al. Real-world comparison of major bleeding risk among non-valvular auricular fibrillation patients initiated on apixaban, dabigatran, rivaroxaban, or warfarin. A propensity score matched analysis. Thromb Haemost. 2016;116:975-86. 